\title{
A comparison of outcomes between a lumen-apposing metal stent with electrocautery-enhanced delivery system and a bi-flanged metal stent for drainage of walled-off pancreatic necrosis
}

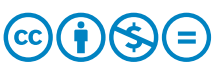

\author{
Authors \\ Oppong ${ }^{1,2}$ \\ Institutions \\ 1 HPB Unit, Freeman Hospital, Newcastle upon Tyne, \\ United Kingdom \\ 2 Department of Gastroenterology, Freeman Hospital, \\ Newcastle upon Tyne, United Kingdom \\ 3 Department of Surgery, Freeman Hospital, Newcastle \\ upon Tyne, United Kingdom \\ 4 Department of Gastroenterology, St. James's University \\ Hospital, Leeds, United Kingdom
}

Noor L. H. Bekkali ${ }^{1,2}$, Manu K. Nayar ${ }^{1,2}$, John S. Leeds ${ }^{1,2}$, Richard M. Charnley²,3, Matthew T. Huggett ${ }^{4}$, Kofi W.

submitted 2.6.2017

accepted after revision 31.7.2017

Bibliography

DOI https://doi.org/10.1055/s-0043-120831 |

Endoscopy International Open 2017; 05: E1189-E1196

(c) Georg Thieme Verlag KG Stuttgart · New York

ISSN 2364-3722

Corresponding author

Kofi W. Oppong, MD, HPB Unit, Freeman Hospital, Newcastle upon Tyne NE7 7DN, United Kingdom

Fax: +44-191-2231249

Kofi.oppong@nuth.nhs.uk

\section{ABSTRACT}

Background and study aims Bi-flanged metal stents (BFMS) have shown promise in the drainage of walled-off pancreatic necrosis (WON), but their placement requires multiple steps and the use of other devices. More recently, a novel device consisting of a combined lumen-apposing metal stent (LAMS) and electrocautery-enhanced delivery system has been introduced. The aim of this study was to compare the placement and outcomes of the two devices. Patients and methods This was a retrospective review of consecutive patients undergoing endoscopic ultrasoundguided placement of BFMS or LAMS for drainage of symptomatic WON. Data from procedures between October 2012 and December 2016 were taken from a prospectively maintained database. We compared technical and clinical success, procedure time, costs, and composite end point of significant events (adverse events, stent migration, additional percutaneous drainage) between BFMS and LAMS.

Results 72 consecutive patients underwent placement of BFMS (40 patients, 44 stents) or LAMS (32 patients, 33 stents). Technical success was $91 \%$ for BFMS and $97 \%$ for LAMS. Clinical success was $65 \%$ vs. $78 \%$, respectively. Median in-room procedure time was significantly shorter in the LAMS group (45 minutes [range $30-80$ ]) than in the BFMS group (62.5 minutes [range $35-135$ ]; $P<0.001)$ and fewer direct endoscopic necrosectomies (DEN) were performed (median $1[0-2.0]$ vs. $2[0-3.7]$, respectively; $P=0.005$ ). If only inpatients were considered (35 BFMS and 19 LAMS), there was no significant difference in DEN 2 (range 0 - 11) and 2 (range $0-8$ ), respectively. The composite end point of $32 \%$ vs. $24 \%$ was not significantly different. Median procedural costs for all patients with successful stent placement for WON treatment was $€ 4427$ (range 1630 - 12926) for BFMS vs. $€ 3500$ (range 2509-13 393) for LAMS ( $P=$ $0.10)$.

Conclusion LAMS was superior to BFMS in terms of procedure time, with comparable adverse events, success, and costs.

\section{Introduction}

Minimally invasive techniques, including endoscopic ultrasound (EUS)-guided cystgastrostomy, are the preferred options for the management of pancreatic fluid collections including symptomatic walled-off necrosis (WON) occurring as sequelae of severe acute pancreatitis [1,2]. Recently, purpose-designed stents have become available for cystgastrostomy, and one of the first such stents was the bi-flanged metal stent (BFMS). These stents have shown promise in the drainage of pancreatic fluid collections, and WON specifically, but with a significant migration/dislodgment rate in initial studies [3,4]. In addition, the BFMS still requires multiple steps and the use of other devi- 
ces to gain access to the cavity, dilate the tract prior to insertion, and deploy the stent.

A shorter stent with more defined lumen-apposing features has been developed [5-8]. In its first iteration this stent required the same multi-step procedure as for placement of BFMS stents. Further refinement of the stent delivery system of this lumen-apposing metal stent (LAMS) has produced a secondgeneration device that incorporates an electrocautery tip. This enables a single device to be used when previously multiple devices and steps were required. The literature on the use of this device is limited to a few multicenter registry studies $[9,10]$. The second-generation LAMS is considerably more expensive than previous devices, and studies comparing BFMS with the new-generation, single-step LAMS are lacking.

The aim of this study therefore was to compare placement and outcomes of BFMS and LAMS for the drainage of WON.

\section{Patients and methods}

\section{Study design}

Our center provides a regional multidisciplinary service for severe acute pancreatitis. Patients in outlying hospitals who require minimally invasive endoscopic or surgical intervention for WON are transferred to our unit for management. Decision making and selection of intervention modality are made by the multidisciplinary team after clinical assessment and review of imaging. This study was a retrospective review of consecutive patients undergoing EUS-guided drainage or debridement of symptomatic WON between October 2012 and December 2016, utilizing a prospectively maintained database.

Ethical approval from an institutional review body was not required for this study. Data collection was performed as part of ongoing quality assessment of our service, and the appropriate institutional authorization (Caldicott approval) to hold the patient database for use for quality improvement was obtained. All aspects of the study were conducted in accordance with the Declaration of Helsinki 1964, as revised in Tokyo 2004. Written informed consent was obtained from all patients prior to the procedure.

\section{Patients}

All patients with symptomatic WON undergoing EUS-guided cystgastrostomy were included in the study.

\section{Procedures}

All patients underwent endoscopy with a linear echoendoscope under general anesthesia or conscious sedation. Patients were administered broad-spectrum antibiotics during the procedure. All procedures were performed by endosonographers with extensive experience in the endoscopic management of pancreatic fluid collections.

A therapeutic Pentax echoendoscope (EG-3870UTK; Pentax, Tokyo, Japan) and Hitachi ultrasound workstation (EUB 7500, HI Vison Preirus; Hitachi Medical Corp., Tokyo, Japan) were used. EUS imaging with Doppler flow guidance was used to assess local vasculature and to determine the cyst size, necrosis, and puncture site (transgastric or transduodenal).
Between October 2012 and December 2015, the BFMS was the only specifically designed metal stent available in the UK and was used exclusively in our unit to drain WON. After December 2015, the LAMS stent was also available. Between December 2015 and March 2016, both BFMS and LAMS were used, and choice of stent was dependent on availability. In March 2016, the cystotome that we used as part of the BFMS procedure became unavailable due to production problems. From that point, LAMSs were exclusively used for first WON drainage.

\section{Placement of BFMS}

The puncture site was either transgastric or transduodenal, as previously described, using a cystotome (Cook CST-10; Cook Medical, Winston-Salem, North Carolina, USA) [4]. A 0.035inch guidewire (Jagwire; Boston Scientific, Marlborough, Massachusetts, USA) was inserted into the cavity through the cystotome. The cystotome was then removed and the tract was dilated using a dilation balloon (Cook Medical) to $6-10 \mathrm{~mm}$, if considered necessary by the endoscopist. The fully covered biflanged stent (NAGI; Taewoong Medical, Gyenoggi-do, Korea) was then passed over the wire, and positioned and deployed under fluoroscopic and endoscopic guidance. Stents were of 14 or $16 \mathrm{~mm}$ in diameter and were 20 or $30 \mathrm{~mm}$ in length. Stent size was decided by the endosonographer at the time of the procedure. No lavage or debridement was performed at the index endoscopy.

\section{Placement of LAMS}

The puncture site was either transgastric or transduodenal. The collection was punctured under EUS control using the electrocautery wire at the tip of the device (Hot AXIOS; Boston Scientific). Once the device was satisfactorily positioned within the WON, the distal flange of the stent was deployed under EUS control. The device was then pulled back until the distal flange deformed against the cavity wall. The proximal flange was then deployed on the luminal side either under direct endoscopic control or EUS guidance according to endosonographer preference. In all cases, $15 \times 10 \mathrm{~mm}$ stents were used. During initial experience the deployed stent lumen was dilated at the discretion of the endoscopist. No lavage or debridement was performed at the index procedure.

\section{Direct endoscopic necrosectomy}

After the index procedure, some patients required direct endoscopic necrosectomy (DEN). The decision to perform DEN was taken by the multidisciplinary pancreatitis team. Subsequent decisions to place plastic stents through the existing metal stent were left to the discretion of the endoscopist and were not subject to protocol.

\section{Stent removal}

Stent removal was not to protocol but dependent on the assessment of the team managing the individual patient. 


\section{Outcomes}

The primary aim of this study was to compare technical and clinical success. Secondary aims included total in-room procedure time, cost, stent-related adverse events, number of necrosectomies, and need for additional percutaneous drainage among patients with WON drained with BFMS or LAMS.

Technical success was defined as appropriate stent position within the WON on an intention-to-stent basis per device used. Clinical success was defined as clinical improvement without requirement for further DEN and with significant improvement of the WON on cross-sectional imaging $\leq 4$ weeks following stent insertion.

The procedure time was collated for patients undergoing the procedure under conscious sedation in the endoscopy room. Procedures performed in the operation room under general anesthesia were excluded. Procedure time was defined as the time from patient entry into the endoscopy room to patient departure. This total time included patient and equipment preparation time, time needed to sedate the patient, time needed to complete stent insertion, and the time needed to prepare the patient to leave the endoscopy room.

\section{Costs}

To enable appropriate comparison, patients who underwent stent placement as day-case procedures and those who died $\leq 7$ days after stent placement were excluded from cost analysis.

Procedural costs included device costs based on the stents and equipment needed to deploy the stents (e.g. cystotome, dilation balloon), additional plastic stents, and the costs of any subsequent endoscopic necrosectomy. For the index procedure, the endoscopy cost(s) was not included as all procedures incurred this cost, but it was included for subsequent necrosectomies. The cost of the LAMS used in our hospital was $€ 2405$ and the cost of BFMS, including all additional devices required for its deployment, was $€ 1562$.

Total costs included device costs, necrosectomy procedural costs, and costs for hospital stay. Costs for hospital stay were calculated as total stay after stent insertion until discharge, multiplied by the bed-day tariff as used by the English National Health Service (NHS) [11].

\section{Stent complications}

Stent deployment failure was recorded when the WON was punctured but the procedure did not progress to stent deployment or when the procedure was deemed too technically challenging to attempt after EUS assessment had been performed.

A stent was defined as misplaced when the cavity was punctured but with subsequent incorrect placement of the stent into the cavity or into the stomach.

Stent dislodgment was defined as dislodgment due to manipulation, such as during DEN or during plastic stent removal across the BFMS or LAMS.

Stent migration was recorded when stents were no longer in situ at planned removal or interval scan. Stent migration was defined according to Lakhtakia et al. [12]:
- clinically insignificant - spontaneous external migration of stent after complete resolution of WON

- clinically significant

- internal migration - migration of the stent into the cavity, with or without persistent WON

- external migration - migration of the stent with persistent WON.

\section{Adverse events}

Immediate procedure-related adverse events (e.g. bleeding, perforation or stent misplacement) were defined as adverse events occurring at the index procedure.

The 30-day adverse events were defined as adverse events, such as stent dislodgment at DEN, clinically significant stent migration, perforation or bleeding at DEN, procedure-related death, that occurred within 30 days.

The composite end point comprised any adverse event within 30 days and additional percutaneous drain.

\section{Hospital stay}

The hospital stay was calculated from the day of stent insertion to discharge from the hospital for patients who had BFMS or LAMS placement as an inpatient. To determine the effect of each stent on subsequent procedures, deaths $\leq 7$ days of stent placement were excluded from this analysis.

\section{Statistical analysis}

Patient data were analyzed using MedCalc software, version 11.2.1.0 (MedCalc Software, Ostend, Belgium) and GraphPad prism, version 7 (Graphpad Software Inc., La Jolla, California, USA). The Mann-Whitney $U$ two-tailed test was applied to calculate differences between groups when data were nonparametric, and presented as median and range or $95 \%$ confidence intervals $(\mathrm{Cl})$. The Student's $t$ test was applied for parametric data and presented as mean $(95 \% \mathrm{Cl})$. For categorical data, Fisher's exact or chi-squared test was used. Statistical significance was determined at $P \leq 0.05$. A retrospective power calculation of the sample size required to identify a $13 \%$ difference $(65 \%$ vs. $78 \%)$ in clinical success was performed $(\alpha=0.05, \beta=0.20)$; this was 189 in each group.

\section{Results}

A total of 77 consecutive procedures were attempted in 72 patients. Overall, 44 BFMS and 33 LAMS were inserted. Median age was 63 years (range 11-81 years) in the BFMS group and 57 years (range $19-81$ years) in the LAMS groups $(P=0.94)$. Significantly more procedures were performed as day cases in the LAMS group $(13 / 32 ; 40.6 \%)$ compared with the BFMS group $(5 / 40 ; 12.5 \% ; P=0.01)$. Other baseline characteristics are given in $>$ Table 1 .

Additional percutaneous drainage was performed in three BFMS and four LAMS patients $(P=0.70)$, and surgical debridement was needed in two patients in the BFMS group ( $\triangleright$ Table 2 ). Double-pigtail stents were placed in 13 BFMS patients compared with 7 LAMS patients $(P=0.43)$. One patient from the BFMS 
- Table 1 Baseline characteristics for patients in whom placement of either a bi-flanged metal stent or a lumen apposing stent was attempted for walled-off pancreatic necrosis.

\begin{tabular}{|c|c|c|c|}
\hline & BFMS $(n=40)$ & LAMS $(n=32)$ & $P$ value $^{1}$ \\
\hline Age, median (range), years & $63(11-81)$ & $57(19-81)$ & 0.94 \\
\hline Male, n (\%) & $27(67.5)$ & $18(56.3)$ & 0.34 \\
\hline \multicolumn{4}{|l|}{ Aetiology, $\mathrm{n}$} \\
\hline - Gallstone & 33 & 20 & 0.81 \\
\hline - Alcohol related & 1 & 3 & 0.32 \\
\hline " Other/unknown & 6 & 9 & 0.24 \\
\hline Size of WON, mean $(95 \% \mathrm{Cl}), \mathrm{cm}$ & $14(11-17)$ & $15(7.7-21.7)$ & 0.85 \\
\hline Estimated necrosis, median $\%(95 \% \mathrm{Cl})$ & $35(30-50)$ & $40(20-50)$ & 0.16 \\
\hline Time from acute episode to drainage, median $(95 \% \mathrm{Cl})$, weeks & $7.7(5.8-10.8)$ & $10.6(6.0-31.3)$ & 0.10 \\
\hline Day-case procedures, n (\%) & $5(12.5)$ & $13(40.6)$ & 0.01 \\
\hline \multicolumn{4}{|l|}{ Reason for drainage, $\mathrm{n}$} \\
\hline - Sepsis & 23 & 13 & 0.24 \\
\hline - Pain & 2 & 6 & 0.13 \\
\hline - Pain and sepsis & 10 & 3 & 0.12 \\
\hline - Inability to eat adequately & 5 & 9 & 0.14 \\
\hline - Other & 0 & 1 & 0.44 \\
\hline Admission on ICU during hospital stay, n (\%) & $13(32.5)$ & 7 (21.9) & 0.43 \\
\hline
\end{tabular}

$\checkmark$ Table 2 Number of patients needing percutaneous drainage.

\begin{tabular}{|l|c|c|c|}
\hline & BFMS & LAMS & $\boldsymbol{P}^{\text {value }}{ }^{\mathbf{1}}$ \\
\hline Percutaneous drainage prior to EUS & 9 & 7 & $>0.99$ \\
\hline Percutaneous drainage post-EUS & 3 & 4 & 0.70 \\
\hline Surgical debridement & 2 & 0 & 0.50 \\
\hline $\begin{array}{l}\text { BFMS, bi-flanged metal stent; LAMS, lumen-apposing metal stent; EUS, } \\
\text { endoscopic ultrasound. } \\
\text { 1 The chi-squared or Fisher's exact test was applied to calculate the } P \text { value. }\end{array}$
\end{tabular}

group underwent LAMS placement for unsatisfactory drainage with a BFMS.

\section{Technical success}

In the BFMS group, technical success was 90.9\% (40/44 stents in 40 patients). Stent deployment failure occurred in two patients; one subsequently underwent successful transpapillary drainage and the other underwent successful stenting during a subsequent procedure. Both patients met the criteria for clinical success at 4 weeks.

In two other patients, there was stent misplacement with subsequent correct placement, necessitating usage of two stents per patient. Both patients met the criteria for clinical success at 4 weeks.
In the LAMS group, the technical success was $97 \%$ (32/33). In one patient, with a pre-existing LAMS, stent misplacement occurred during drainage of a second noncommunicating collection. Successful stent placement using another LAMS during the same procedure was subsequently achieved; this patient needed DEN beyond 4 weeks. Eight procedures with LAMS had subsequent balloon dilation of the stent during initial experience with this type of stent.

Clinical outcomes following stent insertion are shown in - Table 3.

\section{Clinical success}

Cross-sectional imaging $\leq 4$ weeks was available for $30 / 40$ patients in the BFMS group; 3 patients had follow-up scans $>4$ weeks and 7 died $<4$ weeks or were outpatients. Cross-sectional imaging was available for 18/32 patients in the LAMS group; 2 patients had follow-up scans $>4$ weeks and 12 died $<4$ weeks or were inpatients. All scans showed WON improvement. Clinical success was achieved in 26 BFMS patients (65\%) and 25 LAMS patients $(78 \%)$ at 4 weeks post-drainage $(P=0.29)$.

\section{Procedure time}

Median in-room procedure time for all cases with stent deployment was 62.5 minutes (range 35-135 minutes) for 21/40 BFMS and 45 minutes (range $30-80$ minutes) for 26/33 LAMS $(P<0.001)$. 
- Table 3 Clinical outcomes of bi-flanged metal stents versus lumen-apposing metal stents.

\begin{tabular}{|c|c|c|c|}
\hline & BFMS & LAMS & $P$ value $^{1}$ \\
\hline Patients, n & 40 & 32 & \\
\hline Number of necrosectomy procedures, median $(95 \% \mathrm{Cl})$ & $2.0(2.0-3.7)$ & $1.0(0-2.0)$ & 0.005 \\
\hline Hospital stay post-EUS, median $(95 \% \mathrm{Cl})$, days & $28(17-45)$ & $25(13-45)$ & 0.83 \\
\hline Time between stent insertion and removal, median $(95 \% \mathrm{Cl})$, weeks & $7.8(5.4-14.0)$ & $9.0(6.0-13.6)$ & 0.52 \\
\hline Time between stent insertion and attempted retrieval, ${ }^{2}$ median $(95 \% \mathrm{Cl})$, weeks & $12.2(4.1-43.3)$ & $10.4(2.5-26.3)$ & 0.75 \\
\hline Stents, $\mathbf{n}$ & 44 & 33 & \\
\hline \multicolumn{4}{|l|}{ 30-day adverse events. $\mathrm{n}$} \\
\hline - Stent misplacement & 2 & 1 & $>0.99$ \\
\hline - Stent deployment failure & 2 & 0 & 0.50 \\
\hline - Additional percutaneous drain & 3 & 4 & 0.45 \\
\hline - Dislodged stent during necrosectomy & 5 & 3 & $>0.99$ \\
\hline - Clinically significant stent migration & 2 & 0 & 0.50 \\
\hline Composite end point, $\mathrm{n}(\%)$ & $14(31.8)$ & $8(24.2)$ & 0.61 \\
\hline
\end{tabular}

\section{Costs}

Median procedural costs for all patients with successful stent placement for WON treatment using BFMS were $€ 4427$ (range $1630-12926)$ vs. $€ 3500$ (range $2509-13393)$ for LAMS ( $P=$ $0.10)$.

Median total costs (including device, necrosectomies, and hospital stay) for inpatients who had BFMS placement were $€ 17189$ (range $3518-157342$ ) vs. $€ 18221$ (range 581457298) for LAMS $(P=0.98)$.

\section{Stent complications}

Stent migration was observed in six patients prior to retrieval in the BFMS group compared with seven in the LAMS group $(P=$ 0.54). In the BFMS group, two stent migrations were classified as clinically significant, as further interventions were required for the collection, and four were classified as clinically insignificant. In the LAMS group, all seven patients had clinically insignificant stent migration.

Stent dislodgment during DEN was observed in five cases in the BFMS group and three in the LAMS group.

Three patients from the BFMS group and one from the LAMS group died with the stent in situ. Stent removal was uncomplicated in all attempted BFMS patients. All BFMS stents were removed, dislodged or migrated. In the LAMS group, two stents have yet to be removed. One patient in the LAMS group was initially lost to follow-up and hence, removal attempt was delayed until after 26 weeks, when the stent was found embedded. Further attempts at removal have been postponed because of pregnancy in this patient. Another patient was lost to follow-up.

\section{Adverse events}

Early adverse events, during the index procedure, consisted of two stent deployment failures and two stent misplacements in the BFMS group. In the LAMS group, one stent was misplaced.

In the BFMS group, three patients died from pre-existing multi-organ failure at 2, 3, and 17 days, respectively, post-stent placement.

In the LAMS group, one patient with known thromboembolic disease died due to massive pulmonary embolism 2 days after stent insertion. None of the deaths were stent related.

\section{Direct endoscopic necrosectomy procedures}

Fewer median necrosectomy procedures were performed in the LAMS group (1 [range $0-2.0$ ]) than in the BFMS group (2 [range $2.0-3.7$ ]; $P=0.005$ ). DEN procedures in the two groups are shown in $>$ Table 4.

When the outpatients were excluded from the analysis, comparable median DEN procedures were found between 35 BFMS patients (2 [range $0-11$ ]) and 19 LAMS patients (2 [range $0-8])$.

\section{Length of hospital stay}

Median hospital stay was comparable between the BFMS and LAMS patients: 28 days (range $2-320$ days) and 25 days (range $4-108)$, respectively $(P=0.83)$. 
- Table 4 Cumulative number of patients needing direct endoscopic necrosectomy after bi-flanged metal stent or lumen-apposing metal stent placement.

\begin{tabular}{|c|c|c|c|}
\hline & BFMS $(n=40)$ & LAMS $(n=32)$ & $P$ value $^{1}$ \\
\hline \multicolumn{4}{|c|}{ Time to DEN after stent placement, $\mathrm{n}(\%)$} \\
\hline - $1-<4$ weeks & $25(63)$ & $20(63)$ & $>0.99$ \\
\hline - $4-<8$ weeks & $12(30)$ & $7(22)$ & 0.59 \\
\hline - $\geq 8$ weeks & $7(18)$ & $1(3)$ & 0.14 \\
\hline
\end{tabular}

\section{Discussion}

The results of this study demonstrate that use of a single-device LAMS is associated with a statistically significant shorter procedure time compared with BFMS. Overall, total procedure costs were similar for both systems, and we saw nonsignificant lower adverse events and better clinical outcomes with the use of LAMS. To the best of our knowledge, our study is the first to compare a multiple-step deployment BFMS device with the newly developed LAMS with electrocautery-enhanced delivery system, in the setting of a multidisciplinary center utilizing standardized pathways for management and intervention for severe acute pancreatitis.

Historically, EUS-guided drainage of WON has relied on the use of biliary plastic stents to maintain patency of the cystgastrostomy [13-15]. There has been interest in the use of fully covered, self-expandable, metal stents (FCSEMS) to maintain a larger opening to potentially improve drainage, and initially biliary or esophageal FCSEMS were utilized [16-19]. This is further encouraged by the high success rates previously reported for placing these metal stents $97 \%-100 \%[5-7,12,19,20]$. In our study, technical success was $97 \%$ for LAMS. Although there is as yet no clear-cut evidence of superiority over plastic stents $[21,22]$, metal stent technology has rapidly advanced, with several competing designs. Postulated advantages of the second-generation LAMS inherent in its design are shorter procedure duration, reduced risk of immediate procedure-related adverse events, and reduced risk of misplacement and dislodgment of the stent compared with the first-generation LAMS or BFMS. However, these advantages are as yet unproven. The potential benefits of the LAMS device relate to two main aspects. First, only one integrated device is used, abolishing the need to exchange devices over a wire and dilate the tract prior to stent placement. The duration of the procedure should therefore be shorter, and the technical difficulty and the risk of adverse events at the index procedure may be reduced. Second, the short length and lumen-apposing features of the stent may reduce the risk of dislodgment and migration. Our data confirm that a single-device stent is more time efficient than the multiple-step system, with a median reduction of patient "in-room time" of 20 minutes per case, a significant saving in expensive endoscopy room time, potentially enabling more procedures; for example, a diagnostic gastroscopy could be performed in this time (NHS tariff €355) [23]. In addition, although the dif- ference in technical success (6\%) between the stents did not reach statistical significance, it is of potential clinical relevance. The one difference in baseline characteristics between the two groups was the number of day-case procedures, which were significantly more common in the LAMS group $(40.6 \%$ vs. $12.5 \%$ ). Day-case patients are likely to have less-severe disease than inpatients and therefore less likely to require DEN. Overall, fewer necrosectomy procedures were needed in LAMS patients; however, this difference disappeared when the analysis excluded ambulatory care patients, suggesting comparable drainage between the two stents.

Stent migration is clinically relevant when the stent dislodges before resolution of the WON, as this may result in sepsis and the need for further intervention. However, a stent migration that occurs after WON resolution may have no clinical impact, and could be regarded as beneficial as it precludes tissue overgrowth of the stent and removes the need for a procedure to retrieve the stent. It has been suggested that it should be categorized separately [12]. Stent migration is defined in a variety of ways in different studies, with some classifying dislodgment during DEN and spontaneous migration regardless of clinical relevance, whereas other studies have chosen to separate dislodgment and migration. In an Australian multicenter study utilizing the same BFMS as that used in the present study, an overall migration rate of $20.4 \%$ was reported [3]. In our study, BFMS migration occurred in $6 / 40$ patients (15\%), but of these, only two were clinically significant, giving a clinically relevant migration rate of $5 \%$. In comparison, LAMS migration occurred in seven patients, and was clinically insignificant in all cases. Dislodgment, which in our study occurred in $12.5 \%$ of BFMS patients and $9 \%$ of LAMS patients, is best reported separately as this is dependent not only on the characteristics of the stent but also on the DEN technique used. The BFMS dislodgment rate is comparable to that reported previously [20]. The largest BFMS study to date followed a strict protocol for endoscopic intervention after stent placement and showed a $0 \%$ stent migration rate [12].

Although the delivery system for first- and second-generation LAMS is very different, the dimensions, material, and physical properties of the stents are identical and would therefore be expected to exhibit the same characteristics and properties once in situ. A number of previous studies of first- and secondgeneration LAMS have reported technical success rates of 
$97 \%-100 \%$, clinical success of $86 \%-93 \%$, and migration rate of $0-6 \%[5,6,8]$. A recent nationwide Spanish study of FCSEMS for drainage of pancreatic fluid collections found $97 \%$ technical success and $85 \%$ clinical success [19]. This study included 73 LAMS, which had a $4 \%$ stent migration rate [19]. The number of stent migrations without clinical impact observed in the current study may be due to the fact that we did not follow a strict protocol to remove stents at a pre-determined time; rather, removal was based on clinical response combined with computed tomography findings of resolution of WON.

Overall, the composite end point rate was lower in the LAMS group than the BFMS group, although the difference was not statistically significant. A recent study, using the previous generation LAMS (AXIOS; Boston Scientific), showed superiority of LAMS over plastic stents but with more adverse events [8]. In contrast, in our study of the second-generation device, the adverse event rate (utilizing the same definitions of bleeding, perforation, and misplacement) was low (3\%), and other studies of the device have also reported very low procedural adverse events $[9,10]$. A possible explanation is that placement of the first-generation device requires the same multiple steps and therefore potential for adverse events as other metal and plastic stents. Conversely, a recent report from an ongoing randomized controlled trial of the second-generation LAMS and plastic stents for drainage of WON reported a very high rate of stent-related bleeding for LAMS ( $n=3 / 12 ; 25 \%) 124$. However, other larger registry-based studies have not reported a similar problem $[9,10,25]$, and neither did our series, with fewer immediate adverse events. The results of the completed study are awaited.

Although the LAMS device is significantly more expensive than BFMS, we found no significant difference between the two stents in total procedural costs for WON treatment.

There are several limitations to our study owing to its retrospective nature. Stent selection was not randomized but rather represented availability over two consecutive time periods. In addition, although we found no significant difference in baseline characteristics between the two groups, it is possible that one group contained patients with more severe pancreatitis, although this would not impact on our principal findings of reduced procedure duration and comparable costs. Finally, when we performed a retrospective power calculation, we found that the study was underpowered for this sized difference. A strength of the study is that it was performed at a single center with a consistent multidisciplinary approach to the management of WON during the duration of the study.

Comparisons between different studies in this field are complicated by the lack of consistent definitions for outcomes and adverse events, and the heterogeneity of the fluid collections considered. In addition, even when comparison is restricted to WON, the diversity of the patient group in terms of quantity of necrotic material, maturation of the collection, severity of pancreatitis, and clinical condition at the time of drainage, makes comparisons between studies problematic.

In conclusion, this study suggests that the novel single-device LAMS, though a more expensive device, is superior in terms of procedure time, with comparable costs and composite end point to multi-step BFMS. A head-to-head randomized controlled trial would be required for definitive conclusions.

\section{Competing interests}

Dr. Huggett has received Lecturing fees from Olympus. Lecturing fees and travel bursaries from Boston, travel bursaries from Cook, Aquilant Medical and Norgine. Dr. Bekkali has received a travel grant from Boston Scientific and from Cook Medical. Dr. Nayar, Dr. Haugk and Dr. Oppong have received speaker fees from Medtronic. Dr. Oppong received a travel grant from Medtronic. All other authors disclose no financial relationships relevant to this article.

\section{References}

[1] Bakker OJ, van Santvoort HC, van Brunschot S et al. Endoscopic transgastric vs surgical necrosectomy for infected necrotizing pancreatitis: a randomized trial. JAMA 2012; 307: 1053-1061

[2] Varadarajulu S, Bang JY, Sutton BS et al. Equal efficacy of endoscopic and surgical cystogastrostomy for pancreatic pseudocyst drainage in a randomized trial. Gastroenterology 2013; 145: 583 - 590.e1

[3] Chandran S, Efthymiou M, Kaffes A et al. Management of pancreatic collections with a novel endoscopically placed fully covered self-expandable metal stent: a national experience (with videos). Gastrointest Endosc 2014; $2: 1$ - 9

[4] Huggett MT, Oppong KW, Pereira SP et al. Endoscopic drainage of walled-off pancreatic necrosis using a novel self-expanding metal stent. Endoscopy 2015; 47: $929-932$

[5] Sharaiha RZ, Tyberg A, Khashab MA et al. Endoscopic therapy with lumen-apposing metal stents is safe and effective for patients with pancreatic walled-off necrosis. Clin Gastroenterol Hepatol 2016; 14: $1797-1803$

[6] Walter D, Will U, Sanchez-Yague A et al. A novel lumen-apposing metal stent for endoscopic ultrasound-guided drainage of pancreatic fluid collections: a prospective cohort study. Endoscopy 2015; 47: $63-67$

[7] Siddiqui AA, Adler DG, Nieto J et al. EUS-guided drainage of peripancreatic fluid collections and necrosis by using a novel lumen-apposing stent: a large retrospective, multicenter U. S. experience (with videos). Gastrointest Endosc 2016; 83: 699-707

[8] Siddiqui AA, Kowalski TE, Loren DE et al. Fully covered self-expanding metal stents versus lumen-apposing fully covered self-expanding metal stent versus plastic stents for endoscopic drainage of pancreatic walled-off necrosis: clinical outcomes and success. Gastrointest Endosc 2017; 85: $758-765$

[9] Rinninella E, Kunda R, Dollhopf M et al. EUS-guided drainage of pancreatic fluid collections using a novel lumen-apposing metal stent on an electrocautery-enhanced delivery system: a large retrospective study (with video). Gastrointest Endosc 2015; 82: 1 -8

[10] Venkatachalapathy S, Makin A, Pereira S et al. OC-073 The first multicentre experience from the UK and Ireland of the use of the Hot Axios System for transluminal drainage of pancreatic fluid collections. Gut 2016; 65: A44

[11] Shadbolt N, O’Hara K, Berners-Lee T et al. Linked open government data: Lessons from data.gov.uk. IEEE Intell Syst 2012; 27: 16-24

[12] Lakhtakia S, Basha J, Talukdar R et al. Endoscopic 'step-up approach' using a dedicated bi-flanged metal stent reduces the need for direct necrosectomy in walled-off necrosis (with videos). Gastrointest Endosc 2017; 85: $1243-1252$ 
[13] Seifert H, Wehrmann T, Schmitt T et al. Retroperitoneal endoscopic debridement for infected peripancreatic necrosis. Lancet 2000; 356: $653-655$

[14] Charnley RM, Lochan R, Gray H et al. Endoscopic necrosectomy as primary therapy in the management of infected pancreatic necrosis. Endoscopy 2006; 38: 925 - 928

[15] Seewald S, Ang TL, Richter $\mathrm{H}$ et al. Long-term results after endoscopic drainage and necrosectomy of symptomatic pancreatic fluid collections. Dig Endosc 2012; 24: 36-41

[16] Talreja JP, Shami VM, Ku J et al. Transenteric drainage of pancreaticfluid collections with fully covered self-expanding metallic stents (with video). Gastrointest Endosc 2008; 68: 1199-1203

[17] Penn DE, Draganov PV, Wagh MS et al. Prospective evaluation of the use of fully covered self-expanding metal stents for EUS-guided transmural drainage of pancreatic pseudocysts. Gastrointest Endosc 2012; 76: 679-684

[18] Weilert F, Binmoeller KF, Shah JN et al. Endoscopic ultrasound-guided drainage of pancreatic fluid collections with indeterminate adherence using temporary covered metal stents. Endoscopy 2012; 44: 780 783

[19] Vazquez-Sequeiros E, Baron TH, Pérez-Miranda M et al. Evaluation of the short- and long-term effectiveness and safety of fully covered self-expandable metal stents for drainage of pancreatic fluid collec- tions: results of a Spanish nationwide registry. Gastrointest Endosc 2016; 84: 450-457.e2

[20] Chandran S, Efthymiou M, Kaffes A et al. Management of pancreatic collections with a novel endoscopically placed fully covered self-expandable metal stent: a national experience (with videos). Gastrointest Endosc 2015; 81: $127-135$

[21] Bang JY, Hawes R, Bartolucci A et al. Efficacy of metal and plastic stents for transmural drainage of pancreatic fluid collections: a systematic review. Dig Endosc 2015; 27: 486 - 498

[22] Gurusamy KS, Belgaumkar AP, Haswell A et al. Interventions for necrotising pancreatitis. Cochrane Database Syst Rev 2016; 4: CD011383

[23] NHS Improvement and NHS England. NHS Improvement and NHS England. 2017: Available from: https://improvement.nhs.uk/uploads/documents/Copy_of_Annex_A_-_National_tariff_workbook. $\mathrm{xlsx}$

[24] Bang JY, Hasan M, Navaneethan U et al. Lumen-apposing metal stents (LAMS) for pancreatic fluid collection (PFC) drainage: may not be business as usual. Gut 2016: doi:10.1136/gutjnl-2016-312812

[25] Leeds JS, Nayar MK, Charnley RM et al. Lumen-apposing metal stents for pancreatic fluid collection drainage: may not be business as usual? Gut 2017; 66: 1530 